(1)

CrossMark

\title{
Phenotyping primary spontaneous pneumothorax
}

\author{
José M. Porcel \\ Affiliation: Pleural Medicine Unit, Dept of Internal Medicine, Arnau de Vilanova University Hospital, IRBLleida, \\ Lleida, Spain. \\ Correspondence: José M. Porcel, Pleural Medicine Unit, Dept of Internal Medicine, Arnau de Vilanova \\ University Hospital, Avda Alcalde Rovira Roure 80, 25198 Lleida, Spain. E-mail: jporcelplayahoo.es
}

@ERSpublications

In the era of personalised medicine, there is a need to "phenotype" primary spontaneous pneumothorax in order to tailor the most appropriate treatment for each patient http://ow.ly/slNX30lizXz

Cite this article as: Porcel JM. Phenotyping primary spontaneous pneumothorax. Eur Respir J 2018; 52: 1801455 [https://doi.org/10.1183/13993003.01455-2018].

Spontaneous pneumothorax is one of the most common disorders affecting the pleura. A large epidemiological study in France from 2008 to 2011 reported an estimated annual rate of non-traumatic pneumothoraces of 22.7 cases per 100000 inhabitants, with a male to female ratio of 3.3 to 1 [1]. $85 \%$ of the nearly 60000 hospital admission episodes corresponded to primary spontaneous pneumothoraces (PSP) [1], a categorisation which implies that the person does not have a known lung disease. Despite spontaneous pneumothorax being frequent and first recognised as a distinct entity two centuries ago [2], only about 20 randomised controlled trials have been published to date, the vast majority of which comprised a small number of patients. Consequently, gaps still remain in the fundamental pathophysiological mechanisms and preferred management options. For instance, the assumption that the underlying lung is normal in PSP is debatable. PSP predominantly ( $90 \%)$ occurs in tobacco smokers [3]. Airway inflammation is produced by tobacco and cannabis smoking and, thus, both contribute to the development of subclinical lung disease, particularly upper lobe bullous emphysema [3]. The general consensus is that rupture of subpleural blebs or bullae (usually located in the lung apices) into the pleural space plays a major role in PSP development. Most patients with a PSP who are thought to be free of parenchymal disease are found to have blebs, bullae (termed emphysema-like pulmonary changes) or apical opacities (i.e. linear opacities, focal subpleural consolidations or pleural thickening) on high-resolution computed tomography (HRCT) [4-6]. Abnormal regions of the visceral pleura detected by fluorescein-enhanced autofluorescence thoracoscopy, so-called "pleural porosity", have also been put forward as a potential cause to explain the occurrence of PSP [7]. However, pleural porosity, which hypothetically implies slow air leakage through the visceral pleura, is difficult to reconcile with the sudden start of PSP symptoms. According to the previous text, the distinction between PSP and secondary spontaneous pneumothorax is viewed increasingly as artificial. In the same line, the need to routinely obtain an HRCT to detect an underlying occult disease for clinical decision-making purposes during a first episode of a PSP should be considered, though not currently explicitly recommended by scientific guidelines [7-9]. In fact, HRCT imaging in patients presenting with an apparent PSP may be cost-effective even if only for the uncovering of infrequent diffuse cystic lung diseases, such as lymphangioleiomyomatosis, Birt-Hogg-Dubé syndrome or Langerhans cell histiocytosis [10]. Spontaneous pneumothorax occurs in around 60\% [11], 75\% [12] and 15\% [13] of these entities, respectively, albeit with a very high likelihood of recurrence [14].

Other critical questions, with so far elusive answers, that may help optimise PSP management are the true risk of recurrences after a first episode and the identification of patients at this particular risk who could benefit from early preventive measures. The study by WALKER et al. [15] in this issue of the European 
Respiratory Journal gives insight into these questions. They performed a meta-analysis of 29 studies (of which only four were randomised controlled trials) comprising more than 13500 adult patients with a first episode of PSP who underwent medical management (i.e. observation, needle aspiration or chest drain). The overall recurrence rate (either ipsilateral or contralateral) was $32 \%$, and at the 1 year mark it was $29 \%$. Patient follow-up varied dramatically among studies (3 to 144 months). In another recently published systematic review that included 40 studies and 3904 patients with a first episode of PSP, the mean recurrence rate was found to be slightly less $(22 \%)$ and the mean time to first recurrence was 20 months, but subjects who underwent surgical interventions were also included [16].

A relevant finding of the WALKER et al. [15] study was that recurrence rates were significantly higher in women than men [15]. A possible reason might be the presence of some female-specific disease, such as catamenial pneumothorax (CP). A retrospective Japanese database identified 27716 women who needed hospitalisation for spontaneous pneumothorax during a 6-year period [17]. Underlying lung diseases were reported in one-third of the cases, CP representing the fourth leading cause (873 cases), after interstitial pneumonia, lung tumours and chronic obstructive airway diseases. In another retrospective series, CP accounted for $21 \%$ of 51 females younger than 50 years of age with spontaneous pneumothoraces who were surgically treated [18]. Even after pleurodesis through video-assisted thoracoscopic surgery (VATS), $30-40 \%$ of CP patients experience recurrences during the following 2 years $[18,19]$. In addition to the influence of the female gender in PSP recurrences, the other consistent conclusion of the WALKER et al. [15] meta-analysis is the recognition that smoking cessation may be the most important strategy in preventing PSP, having been found to be associated with a 4-fold decrease in the risk of recurrence. Other potential predictors of recurrence such as a low body mass index or the presence of blebs or bullae on chest imaging were less consistently demonstrated, probably due to the significant heterogeneity of the included studies [15].

According to the current meta-analysis, patients with a new diagnosis of PSP should be informed that about one-third will have a recurrent episode, mostly during the next year, and this risk will be greatly increased in females. Nonetheless, the life-long recurrence risk may be higher. Paradoxically, existing guidelines do not recommend preventive procedures until the patient experiences a second ipsilateral PSP event $[7,8]$. Should preventive therapies be reserved for recurrent PSP or should they be applied following the first episode, particularly in high-risk patients such as females? In a network meta-analysis of 10 randomised controlled trials, VATS ranked the highest in preventing recurrences in patients with a first episode of PSP, followed by pleurodesis [20]. In a second study, 181 patients with a first episode of PSP were randomised to receive VATS (plus bullectomy of visible blebs/bullae and mechanical pleurodesis) or conventional chest tube drainage [21]. At the 1-year follow-up, the recurrence rate in the chest tube group was significantly higher at $34 \%$ versus the $13 \%$ recurrence rate in the VATS group. Obviously, the benefits of any surgical procedure for such a low-risk mortality disease like PSP should outweigh the non-benefits. In a large prospective series of 1415 patients with recurrent PSP who were subjected to VATS with talc poudrage and, if necessary, bullectomy, complications occurred in 29 (2\%) patients, of whom $24(1.7 \%)$ had prolonged air leaks [22]. There was no mortality and further recurrences were identified in only 26 (1.9\%) patients. Moreover, needlescopic [23] and uniportal [24] VATS are alternative effective options with potentially less postoperative pain and faster recovery that should be further explored in the future. Even if a preventive intervention is not advised a priori according to prevailing guidelines, should patients experiencing their first PSP episode and being treated with a chest tube for the initial management also undergo chemical pleurodesis to prevent future events?

In the era of personalised medicine, there is a need to "phenotype" PSP (i.e. to define categories according to the pathophysiology, clinical course or patient's occupation) in order to tailor the most appropriate treatment for each patient. Future guidelines should propose, despite the limited existing evidence, the use of diverse therapies for different clinical scenarios. Awareness of an unrecognised lung disease and/or categorisation of patients into a high-recurrence risk group may warrant more aggressive management at the initial PSP presentation. Large prospective randomised controlled trials and cost-effectiveness studies are urgently required to implement the most appropriate management strategies in PSP [25].

Conflict of interest: J.M. Porcel has nothing to disclose.

\section{References}

1 Bobbio A, Dechartres A, Bouam S, et al. Epidemiology of spontaneous pneumothorax: gender-related differences. Thorax 2015; 70: 653-658.

2 Kaya SO, Karatepe M, Tok T, et al. Were pneumothorax and its management known in 15th-century Anatolia? Tex Heart Inst J 2009; 36: 152-153. 
3 Ruppert AM, Perrin J, Khalil A, et al. Effect of cannabis and tobacco on emphysema in patients with spontaneous pneumothorax. Diagn Interv Imaging 2018; 99: 465-471.

4 Kim JT, Oh TY, Chang WH, et al. Natural course of spontaneous pneumothorax without bullae or blebs under high-resolution computed tomography. Thorac Cardiovasc Surg 2014; 62: 505-508.

5 Bintcliffe OJ, Edey AJ, Armstrong L, et al. Lung parenchymal assessment in primary and secondary pneumothorax. Ann Am Thorac Soc 2016; 13: 350-355.

6 Kobayashi NS, Nambu A, Kawamoto M, et al. Pulmonary apical opacities on thin-section computed tomography: relationship to primary spontaneous pneumothorax in young male patients and corresponding histopathologic findings. J Comput Assist Tomogr 2018; 42: 33-38.

7 Tschopp JM, Bintcliffe O, Astoul P, et al. ERS task force statement: diagnosis and treatment of primary spontaneous pneumothorax. Eur Respir J 2015; 46: 321-335.

8 MacDuff A, Arnold A, Harvey J, et al. Management of spontaneous pneumothorax: British Thoracic Society Pleural Disease Guideline 2010. Thorax 2010; 65: Suppl. 2, ii18-ii31.

9 Schnell J, Beer M, Eggeling S, et al. Management of spontaneous pneumothorax and post-interventional pneumothorax: German S3 guideline. Respiration 2018; in press [https://doi.org/10.1159/000490179].

10 Gupta N, Langenderfer D, McCormack FX, et al. Chest computed tomographic image screening for cystic lung diseases in patients with spontaneous pneumothorax is cost effective. Ann Am Thorac Soc 2017; 14: 17-25.

11 Gupta N, Finlay GA, Kotloff RM, et al. Lymphangioleiomyomatosis diagnosis and management: high-resolution chest computed tomography, transbronchial lung biopsy, and pleural disease management. An Official American Thoracic Society/Japanese Respiratory Society Clinical Practice Guideline. Am J Respir Crit Care Med 2017; 196: 1337-1348.

12 Gupta N, Kopras EJ, Henske EP, et al. Spontaneous pneumothoraces in patients with Birt-Hogg-Dubé syndrome. Ann Am Thorac Soc 2017; 14: 706-713.

13 Cooley J, Lee YCG, Gupta N. Spontaneous pneumothorax in diffuse cystic lung diseases. Curr Opin Pulm Med 2017; 23: 323-333.

14 Gupta N. Primary spontaneous pneumothorax: looking beyond the usual. Acad Emerg Med 2018; 25: 470-472.

15 Walker SP, Bibby AC, Halford P, et al. Recurrence rates in primary spontaneous pneumothorax: a systematic review and meta-analysis. Eur Respir J 2018; 52: 1800864

16 Villela MA, Dunworth S, Harlan NP, et al. Can my patient dive after a first episode of primary spontaneous pneumothorax? A systematic review of the literature. Undersea Hyperb Med 2018; 45: 199-208.

17 Hiyama N, Sasabuchi Y, Jo T, et al. The three peaks in age distribution of females with pneumothorax: a nationwide database study in Japan. Eur J Cardiothorac Surg 2018; 54: 572-578.

18 Saito T, Saito Y, Fukumoto KJ, et al. Clinical and pathological characteristics of spontaneous pneumothorax in women: a 25-year single-institutional experience. Gen Thorac Cardiovasc Surg 2018; in press [https://doi.org/10. 1007/s11748-018-0952-8].

19 Bricelj K, Srpčič M, Ražem A, et al. Catamenial pneumothorax since introduction of video-assisted thoracoscopic surgery: a systematic review. Wien Klin Wochenschr 2017; 129: 717-726.

20 Vuong NL, Elshafay A, Thao LP, et al. Efficacy of treatments in primary spontaneous pneumothorax: A systematic review and network meta-analysis of randomized clinical trials. Respir Med 2018; 137: 152-166.

21 Olesen WH, Katballe N, Sindby JE, et al. Surgical treatment versus conventional chest tube drainage in primary spontaneous pneumothorax: a randomized controlled trial. Eur J Cardiothorac Surg 2018; 54: 113-121.

22 Cardillo G, Bintcliffe OJ, Carleo F, et al. Primary spontaneous pneumothorax: a cohort study of VATS with talc poudrage. Thorax 2016; 71: 847-853.

23 Chou SH, Chuang IC, Huang MF, et al. Comparison of needlescopic and conventional video-assisted thoracic surgery for primary spontaneous pneumothorax. Minim Invasive Ther Allied Technol 2012; 21: 168-172.

24 Yang Y, Dong J, Huang Y. Single-incision versus conventional three-port video-assisted surgery in the treatment of pneumothorax: a systematic review and meta-analysis. Interact Cardiovasc Thorac Surg 2016; 23: 722-728.

25 Bintcliffe OJ, Hallifax RJ, Edey A, et al. Spontaneous pneumothorax: time to rethink management? Lancet Respir Med 2015; 3: 578-588. 